\title{
AML presenting as bilateral proptosis with lacrimal glands involvement in a young male
}

\author{
Wani R.M ${ }^{1}$, Rashid $\mathrm{S}^{2}$, Mir $\mathrm{AM}^{3}$, Suraya $\mathrm{S}^{4}$ \\ ${ }^{1}$ Dr Raashid Maqbool Wani, Senior Resident, Department of Ophthalmology, Government Medical College, Srinagar, \\ Jammu and Kashmir (India), ${ }^{2}$ Dr Sabia Rashid, ${ }^{3}$ Dr Adil Majeed Mir, ${ }^{4}$ Dr Saqib Suraya. All are affiliated with \\ Government Medical College, Srinagar, Jammu and Kashmir (India)
}

Address for Correspondence: Dr Raashid Maqbool Wani, H.No. 132 (Doctors Enclave), Green Lane Hafiz Colony, Peerbagh, Srinagar Kashmir (India), 190015. Email address: raashid.maqboolwani@ gmail.com

\begin{abstract}
Acute Myeloid Leukemia (AML) is associated with acquired genetic alterations that result in the replacement of normal marrow elements by relatively undifferentiated blast cells exhibiting one or more types of early myeloid differentiation. Bilateral proptosis with involvement of lacrimal glands and papilledema due to acute myeloid leukemia is presented in this case report. Investigations like complete blood count, peripheral blood film along with bone marrow examination help in reaching the diagnosis early and a timely intervention thereby increasing the survival chances in such patients.
\end{abstract}

Key words: Acute Myeloid Leukemia, Proptosis, Lacrimal Glands, Papilloedema, Chemotherapy.

\section{Introduction}

In Acute Myeloid Leukemia (AML), neoplastic myeloid precursor cells accumulate in the marrow and suppress remaining normal hematopoietic progenitor cells, circulate in the blood and invade the tissues of the body. Most patients present within weeks or a few months of the onset of the symptoms with findings related to anaemia, neutropenia and thrombocytopenia [1]. Proptosis has been reported among the clinical features of AML [2]. The case of acute myeloid leukemia presenting with bilateral proptosis, lacrimal glands involvement with papilloedema is a unique case to the best of our knowledge.

\section{Case Report}

A 16 years old boy Asian Indian in origin presented to our ophthalmic clinic with bilateral proptosis and periorbital swelling (more on the superior aspect). Patient was also suffering from low grade fever and headache with normal visual acuity. Patient was earlier seen by a primary care physician and was referred to our clinic for further treatment. Patient was cachexic and ill looking. It is this appearance which prompted us to look for a systemic illness as a cause of the bilateral

\footnotetext{
Manuscript received: $1^{\text {st }}$ June 2015

Reviewed: $15^{\text {th }}$ June 2015

Author Corrected: $24^{\text {th }}$ June 2015

Accepted for Publication: $7^{\text {th }}$ July 2015
}

proptosis patient was suffering from. A detailed history and examination was undertaken. It was found that he had malaise and general body aches with loss of appetite. On systemic examination there was Splenomegaly and a normal neurological examination. Ophthalmic examination revealed bilateral axial proptosis left more than right (Figure 1), slightly painful extraocular movements with restriction of left abduction, lacrimal glands on both sides were tender and enlarged. No afferent pupillary defect was seen. Fundus examination revealed bilateral Papilloedema. Patients had preauricular lymphadenopathy on both sides with no cervical and axillary lymph nodes involvement. His hemoglobin was $8.2 \mathrm{gm} / \mathrm{dl}$, platelets $50000 / \mathrm{ml}$, total leucocyte count was $38100 / \mathrm{mm}^{3}$. His differential count showed $90 \%$ blasts, $2 \%$ Promyelocytes, 5\% Myelo/Metamyelos, $1 \%$ Neutrophil, $2 \%$ lymphocytes. The contrast enhanced axial magnetic resonance images (MRI) showed leukemic infiltrates occupying the lateral wall of the orbits with left more involved than right, which caused the bilateral proptosis in the patient. Axial MRI also showed invasion of the optic nerves and enlargement of the lacrimal glands on both sides (Figure 2). Coronal MRI also revealed enlarged lacrimal glands (Figure 3). There was no infiltration of any extraocular muscles or sinuses. The bone marrow examination revealed total replacement of bone marrow by blasts $18-20$ microns in size, variable 
Nuclear/Chromatin ratio, pale agranular cytoplasm, 2-3 nucleoli with open chromatin. Some of the blasts showed indented/clefted nucleus. Normal haematopoiesis was markedly suppressed. Flowcytometric analysis revealed CD4, CD13, CD33, CD15, CD117, MPO, CD123, CD11b, CD56, CD45, CD34 and HLA-DR Positive. Correlations with clinical features, peripheral blood findings, marrow morphology and cytogenetic studies were suggestive of AML-M1. After diagnosis, patient was started on Adult AML Protocol $3 / 7$ and received induction chemotherapy of daunorubicin $50 \mathrm{mg} / \mathrm{m}^{2} /$ day and cytosine arabinoside $100 \mathrm{mg} / \mathrm{m}^{2} /$ day along with intrathecal therapy of methotrexate, hydrocortisone and Ara-C. Post induction bone marrow examination revealed marrow in remission. Patient responded well to the treatment, proptosis settled (Figure 4). Meanwhile patient developed left facial nerve palsy for which neurology consultation was sought and MRI was done, no definite lesion was found (Figure 5). Hospital stay was complicated by febrile neutropenia and later developed fungal infection of maxillary sinuses for which antifungals were given and patient responded well. On follow up there was complete remission of proptosis and lacrimal gland enlargement.

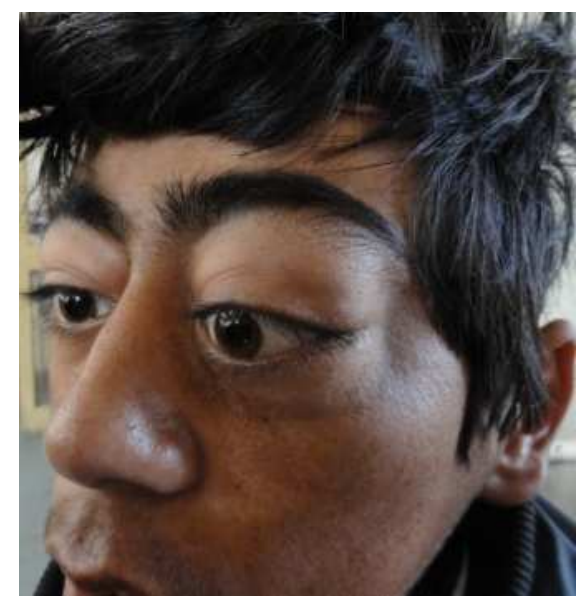

Fig 1: Bilateral axial proptosis left more than right

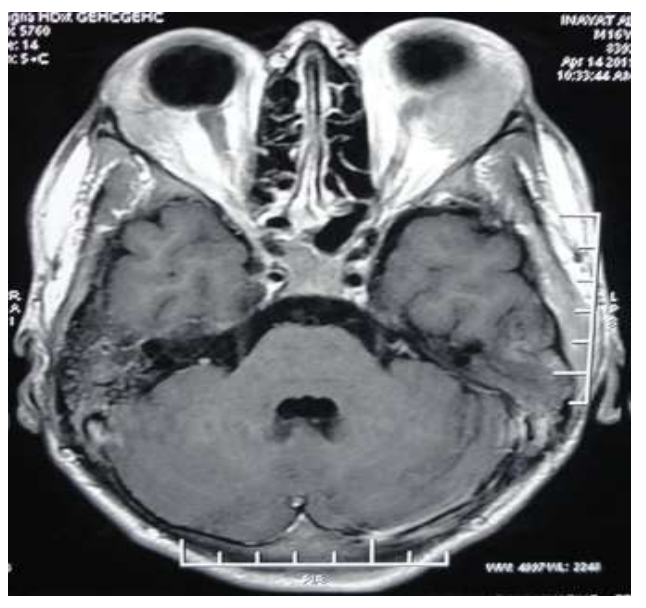

Fig 2: Axial MRI showing invasion of optic nerves

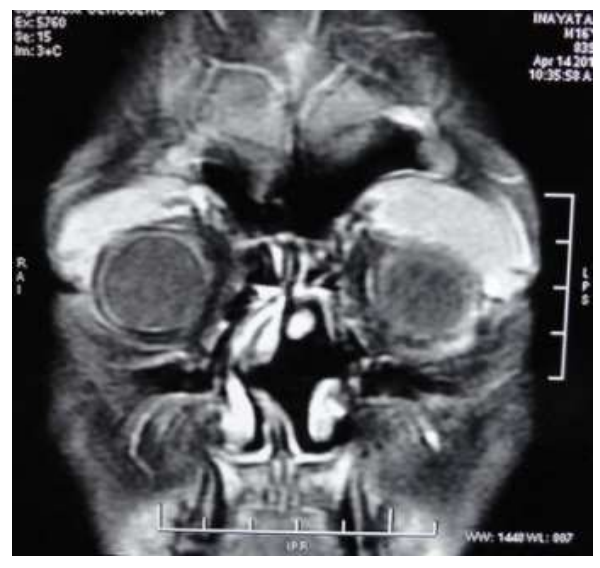

Fig 3: Coronal MRI showing enlarged lacrimal glands (left more than right)

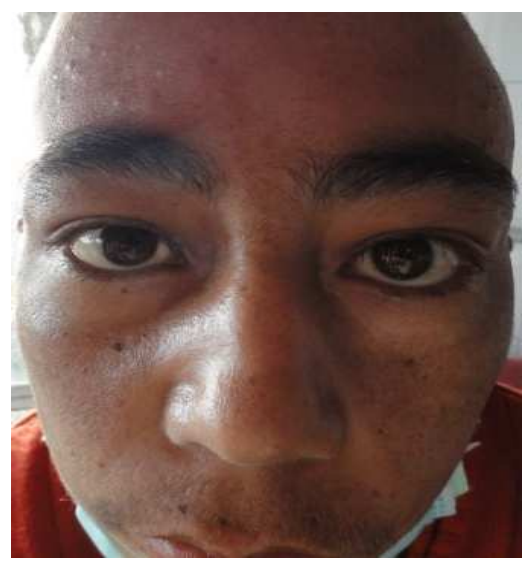

Fig 4: Patient showing resolution of proptosis

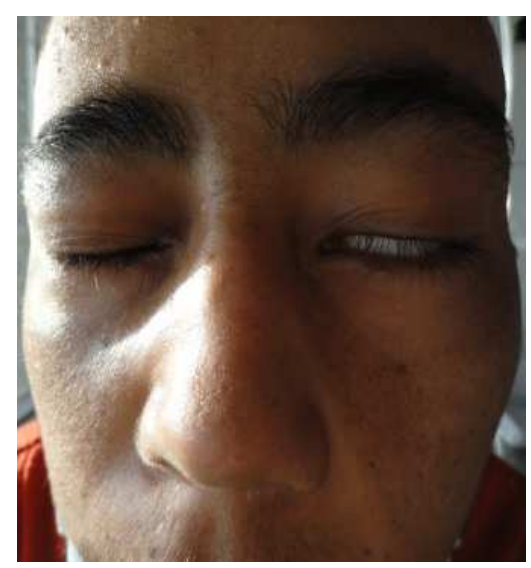

Fig 5: Patient showing left facial nerve palsy post treatment

\section{Discussion}

AML primarily affects adults, peaking in incidence between the ages of 15 and 39 years. It constitutes only $15 \%$ of childhood leukaemia [3]. AML is quite heterogeneous, reflecting the complexities of myeloid cell differentiation. Most patients present with fatigue, fever and spontaneous mucosal and cutaneous bleeding, with thrombocytopenia as the most striking clinical and anatomic feature of the disease. Infiltration of other 
body tissues may lead to lymphadenopathy and splenomegaly. Quite uncommonly (approximately 3\%), patients present with localized masses composed of myeloblasts termed as myeloblastomas, granulocytic sarcomas, or chloromas [4]. Ocular involvement usually occur secondary to leukemic infiltration. Orbital involvement in acute myeloid leukemia often presents with proptosis, most often due to chloromas [5,6,7]. Presence of both unilateral and bilateral proptosis has been reported with AML [8,9,10,11]. Proptosis secondary to diffuse infiltration of the lacrimal gland and infiltration of individual or all extraocular muscles has also been reported [12,13].

Our patient had an unusual presentation of bilateral proptosis with lacrimal glands involvement and bilateral papilloedema. The tumour had infiltrated the lateral walls of the orbit with invasion of the optic nerves and enlargement of the lacrimal glands. The ocular involvement in AML is mainly due to leukemic infiltrates; haemorrhage in the choroid, retina, optic nerve or anterior segment; orbital muscle infiltration or venous blockage. In a study, AML was associated with 9.3\% orbital masses [9]. Other presentations due to orbital involvement include ptosis, lacrimal gland involvement, conjuctival masses, iridic and diffuse uveal involvement $[4,10]$. Most of the reported cases have decreased visual acuity and restricted extra-ocular movements. In our patient, extra-ocular movements were painful with restriction of left abduction but visual acuity was normal. Patient had bilateral periorbital swelling, more on the superotemporal aspect, left more than right. It was firm and tender. Our case was unusual in having simultaneous presence of bilateral proptosis with bilateral papilloedema and lacrimal glands enlargement followed by left facial nerve palsy.

The diagnosis of AML is based on finding greater than $30 \%$ myeloid blasts in the bone marrow. Myeloblasts have delicate nuclear chromatin, two to four nucleoli. The cytoplasm often contains fine, azurophilic, peroxidise positive granules often represented by auer rods. Evaluation of the peripheral smear is an invaluable tool in the diagnosis. Previous reports have highlighted the role of this inexpensive investigation in all cases of childhood proptosis [9, 14]. The peripheral smear reveals the presence of immature blast cells. The total leucocyte count is usually high with a relative neutropenia. Bone marrow examination and flow cytometry should be routinely performed to confirm the diagnosis. Romanowsky stained (Giemsa) air-dried smears are useful in interpreting the hematologic lesions of leukemia and lymphoma. Hematoxylin eosin stain reveals the presence of cells of myeloid lineage with eosinophilic cytoplasmic granules. The prognosis for patients with granulocytic sarcoma depends on the course of the underlying systemic malignancy $[3,10]$. Initiating treatment early can improve the prognosis and outcome. Chemotherapy is the mainstay of treatment. The presence of orbital granulocytic sarcoma does not significantly alter the survival in patients with AML [10]. The rate of remission following chemotherapy also does not seem to be significantly affected due to the presence of granulocytic sarcoma.

\section{Conclusion}

The case of acute myeloid leukemia being presented and having bilateral proptosis, lacrimal glands involvement with papilloedema is a unique case to the best of our knowledge. The classical clinical presentation of AML in the susceptible age group may not be the rule but one should be highly suspicious of the disease if a patient presents with bilateral proptosis and other systemic features as were encountered in our case.

\section{References}

1. Nazik EM, Enaam AA, Ihsan MO et al. Patterns and clinical presentation of acute myeloid leukemia in Sudanese patients. Journal of Science 2015; 5 (1): 23 27.

2. Chen E, Morrison DG, Donahue SP. Acute myeloid leukemia presenting as bilateral proptosis from diffuse extraocular muscle infiltration. Am J Ophthalmol. 2004 May;137(5):948-50.

3. Stein-Wexler R, Wotton-Gorges SL, West DC. Orbital granulocytic sarcoma: an unusual presentation of acute myelocytic leukemia. Pediatr Radiol. 2003 Feb;33(2):136-9. Epub 2002 Nov 12.

4. Stockl FA, Dolmetsch AM, Saornil MA, Font RL, Burnier MN. Jr. Orbital granulocytic sarcoma. Br J Ophthalmol. 1997 Dec;81(12):1084-8.

5. Jha BK, Lamba PA. Proptosis as a manifestation of acute myeloid leukaemia. $\mathrm{Br} \quad \mathrm{J}$ Ophthalmol. 1971 Dec;55(12):844-7. 
6. Puri P, Grover AK. Granulocytic sarcoma of orbit preceding acute myeloid leukemia: a case report. Eur J Cancer Care (Engl). 1999 Jun;8(2):113-5.

7. Swami G, Arya A, Chowdhry B. Acute myeloid leukemia presenting with sudden bilateral proptosis as sole manifestation. Indian Pediatr. 2001 Oct;38(10):1199-200.

8. Zimmerman LE, Font RL. Ophthalmic manifestations of granulocytic sarcoma (myeloid sarcoma or chloroma) Am J Ophthalmol. 1975 Dec;80(6):975-90.

9. Sethi A, Ghose S, Gujral S, Jain P, Kumar R. Childhood proptosis: The invaluable but overlooked peripheral blood smear. Indian J Ophthalmol. 2001 Jun;49(2):121-3.

10. Davis JL, Park DW II, Font RL. Granulocytic sarcoma of the orbit: A histopathologic study. Ophthalmology. 1985 Dec;92(12):1758-62.
11. Rajput $\mathrm{D}^{1}$, Naval R, Yadav K, Tungaria A, Behari S. Bilateral proptosis and bitemporal swelling: A rare manifestation of acute myeloid leukemia. J Pediatr Neurosci. 2010 Jan;5(1):68-71. doi: 10.4103/18171745.66687 .

12. Goldberg L, Tao A, Romano P. Severe exophthalmos secondary to orbital myopathy not due to Graves disease. Br J Ophthalmol. 1982 Jun;66(6):3925.

13. Consul BN, Kulshrestha OP, Mehrotra AS. Bilateral proptosis in acute myeloid leukaemia. $\mathrm{Br} J$ Ophthalmol. 1967 Jan;51(1):65-7.

14. Panda A, Dayal Y: Acute proptosis in myeloid leukaemia. Indian J Ophthalmol. 1984 JulAug;32(4):239-41.

\section{How to cite this article?}

Wani RM, Rashid S, Mir AM, Suraya S. AML presenting as bilateral proptosis with lacrimal glands involvement in a young male. Int J Med Res Rev 2015;3(6):669-672. doi: 10.17511/ijmrr.2015.i6.124. 